\title{
polymers
}

ISSN 2073-4360

www.mdpi.com/journal/polymers

Review

\section{Design of Autonomous Gel Actuators}

\section{Shingo Maeda $^{1 *}$, Yusuke Hara ${ }^{2}$, Satoshi Nakamaru ${ }^{1, \dagger}$ and Shuji Hashimoto ${ }^{2}$}

1 Department of Applied Physics, Graduate School of Science and Engineering, Waseda University, 3-4-1 Okubo Shinjuku-ku, Tokyo 169-8555, Japan;

E-Mail: nakamaru@shalab.phys.waseda.ac.jp (S.N.)

2 Nanosystem Research Institute, NRI, National Institute of Advanced Science and Technology, AIST, Central 5-2, 1-1-1 Higashi, Tsukuba 305-8565, Japan; E-Mails: yhara@aist.go.jp (Y.H.); shuji@waseda.jp (S.H.)

* Author to whom correspondence should be addressed; E-Mail: maeshin@ shalab.phys.waseda.ac.jp; Tel.: +81-3-5286-3233; Fax: +81-3-3202-7523.

$\dagger$ Current Address: Sony Advanced Materials Laboratories.

Received: 15 November 2010; in revised form: 15 December 2010 / Accepted: 10 January 2011 / Published: 11 January 2011

\begin{abstract}
In this paper, we introduce autonomous gel actuators driven by chemical energy. The polymer gels prepared here have cyclic chemical reaction networks. With a cyclic reaction, the polymer gels generate periodical motion. The periodic motion of the gel is produced by the chemical energy of the oscillatory Belouzov-Zhabotinsky (BZ) reaction. We have succeeded in making synthetic polymer gel move autonomously like a living organism. This experimental fact represents the great possibility of the chemical robot.
\end{abstract}

Keywords: gel; gel actuator; chemical robot

\section{Introduction}

Gels have cross-linked networked structures of polymer chains containing solvents; and these gels that swell or shrink due to environmental changes have been studied in various fields [1-5]. Recently, soft robots [6,7] made of electro-active polymer gel [8] have been developed. These gel robots do not need mechanical parts such as motors, drive shafts, gears, etc. because the driving mechanism of the soft robots is different from that of classical mechanical machines. In previous decades, Kachalsky et al. have 
demonstrated an energy conversion system transforming chemical energy into mechanical energy using collagen fibers [8]. Also, since volume phase transition of polymer gels was found [9], many kinds of applications such as a drug delivery system [10], robotic hands [11], and matter transporting device [12], have been studied. The phase transition of polymer gel is induced by hydrogen bonds, coulomb, hydrophobic, and van der Waals interactions. Hence, these devices can operate by controlling external conditions. For example, the poly(N-isopropylacrylamide) (PNIPAAm) is known to undergo a discontinuous volume change on volume change in temperature. In application, dynamic actuation systems by integrating high-aspect-ratio silicon nanocolumns with the PNIPAAm gel have been demonstrated [13]. But, these systems require the complex and fabricated circuit or external control devices because the motion of the polymer gel is driven by external physicochemical signals. On the other hand, in biological systems, there are several autonomous phenomena exhibiting spontaneous motion such as peristaltic motion, heartbeat, brain waves, etc. If such a system is achieved in an artificial system, a novel robot which does not need external control would be expected. However, there are some studies on creating autonomous systems by using chemical energy. Biohybrid actuators from engineered tissue and synthetic polymer thin films have been demonstrated [14]. Although utilization of biopolymer or cell system is one method, our strategy is completely an artificial system.

In this review, we introduce active polymer gel actuators. Gels exhibit a unique capability of undergoing spontaneous volume changes in response to oscillatory chemical reaction. For example, gels can undergo rhythmic swelling and shrinking in response to variations in the $\mathrm{pH}$ of the surrounding solution caused by an oscillating reaction. The $\mathrm{pH}$ oscillations were generated by the Landolt reaction [15] or by an enzymatic reaction [16]. Periodical volume changes were also observed in a $\mathrm{pH}$ responsive gel, which exhibited bistability with diffusion of a reactive substrate into the gel [17]. The chemical reactions were carried out under nonequilibrium conditions provided by precisely controlled continuous-flow stirred tank reactors (CSTR) [15,17]. This means that the condition around the gel is not stationary. From the point of view of engineering, the stationary conditions around the gel are acceptable. So, we focused on the Belousov-Zhabotinsky (BZ) reaction [18]. The BZ reaction is widely known as a chemical oscillating reaction and reaction diffusion system. The overall process is the oxidation of an organic substrate by an oxidizing agent in the presence of a metal catalyst under acidic conditions. Metal ions or metal complexes with high redox potentials, such as cerium ion, ferroin, or ruthenium tris(2,2'-bipyridine) are widely used as catalysts. In the reaction processes, the metal ion periodically changes its charge number to oscillate between the oxidized and reduced states for several hours, as long as the substrate exists. In an unstirred solution, there are chemical waves and spatial pattern formations in the reaction processes. Researchers have developed the mixed system between the polymer and BZ reaction. The use of the polymer gels in the $\mathrm{BZ}$ reaction has so far been confined to eliminating hydrodynamic convection disturbing reaction diffusion patterns [19] and localizing the BZ reaction in a restricted region [20]. In these systems, the structure of the polymer does not change by the BZ reaction because the interaction between the gel structure and the reaction diffusion system is negligibly small. It was reported that polymers and gels undergo a slight spontaneous structure change [21-23]. In the oscillating gel systems, the polymer network itself takes part in the BZ reaction, because the metal catalyst is covalently bounded to the polymer chain. As a result, the reaction medium can change in time and space. In other words, the BZ reaction causes the structural changes of the polymer. When the redox 
state of the metal catalyst moiety in the gel changed, the solubility of the polymer chain changed as well. As a result, the change in the osmotic pressure inside the gel causes the swelling or shrinking of the polymer gel. The displacement of the self-oscillating gel is several dozen micrometers [23]. However, the mechanical displacement of the gel was too small to design the gel actuator. Therefore, in order to construct gel actuators, we should greatly improve displacement of the gel.

\section{Design of Self-Walking Gel}

To create the large deformation of the gel, we introduced a gradient structure into the oscillating gel. To make the gradient structure in the gel, we used the hydrophobic interaction between the $\mathrm{Ru}(\mathrm{bpy})_{3}{ }^{2+}$ moiety and casting mold during polymerization. As a result, a large periodical deformation of the gel coupled with the BZ reaction was achieved [24]. During polymerization, the monomer solution faces two different surfaces of plates: A hydrophilic glass surface and a hydrophobic Teflon surface. Since the $\mathrm{Ru}(\mathrm{bpy})_{3}{ }^{2+}$ monomer is hydrophobic, it is easy to migrate to the Teflon surface side. As a result, a uniform distribution in the direction of the thickness is formed for the component, and the resulting gel has gradient distribution for the content of each component in the polymer network. Thus, the hydrophilic 2-acrylamide-2-methylpropane sulfonic acid (AMPS) component at the glass side was higher than that at the Teflon side. In contrast, the hydrophobic Ru(bpy) ${ }_{3}{ }^{2+}$ moiety at the Teflon side was higher than that at the glass side. Therefore, as for the gel at the AMPS rich side, the swelling ratio was higher than that of the opposite side (i.e., the $\mathrm{Ru}(\mathrm{bpy})_{3}{ }^{2+}$ rich side). Consequently the gel in water bends to the direction of the surface which was faced to the Teflon plate during polymerization. The curvature in the oxidized state was larger than that in the reduced state over the full temperature range. This is because when the hydrophilicity of the polymer increases, the gel expands in the oxidized state. From the deviation of the curvature in the $\mathrm{Ru}(\mathrm{II})$ and the $\mathrm{Ru}(\mathrm{III})$ states, we expected that the gel caused the periodical bending-stretching motion induced by the BZ reaction at constant temperatures.

Figure 1(a) shows the periodical changes of gel motion in the aqueous solution containing the three reactants of the $\mathrm{BZ}$ reaction (malonic acid, sodium bromate and nitric acid) at constant temperatures. The chemical wave evolves in the gel, and it propagates in the direction of the length at constant speed from one edge, attaching the substrate to the other edge. With the propagation of the chemical wave, the distance between the two edges of the gel changes periodically because a spontaneous bending and stretching motion occurs (Figure 1(b)). While the chemical wave exists in the gel $(1 \rightarrow 4)$, the gel stretches. After that, during the reduced state until the next wave appears $(4 \rightarrow 1)$, the gel bends. As shown in Figure 1(c), the displacement of the mechanical oscillation $\left(\Delta l_{\max }\right)$ changes with temperature because the difference in swelling ratio between reduced state and oxidized state depends on temperature. We can see that there is an optimum temperature $\left(18{ }^{\circ} \mathrm{C}\right)$ at which the amplitude becomes the maximum. To convert the bending and stretching changes to the vectorial work, we applied a ratchet mechanism to our gel system. We prepared the ratchet floor with an asymmetrical surface structure as shown in Figure 2(a). On the ratchet floor, the gel repeats bending and stretching autonomously, but sliding backwards is prevented by the teeth of the ratchet. As a result, the gel could move forward. Figure 2(b) shows successive profiles of the "self-walking" motion of the gel in the BZ reaction [25]. The period of chemical oscillation was about $112 \mathrm{~s}$, and the walking velocity of the gel actuator was about $170 \mu \mathrm{m} / \mathrm{min}$. 
Figure 1. (a). Equilibrium swelling ratio expressed as curvature of the poly(NIPAAm-co$\mathrm{Ru}$ (bpy) ${ }_{3}$-co-AMPS) gel strip in cerium sulfate solutions as a function of temperature. Closed square: $\mathrm{Ce}_{2}\left(\mathrm{SO}_{4}\right)_{3}=0.005 \mathrm{M}$ and $\mathrm{HNO}_{3}=0.894 \mathrm{M}$; Open square: $\mathrm{Ce}\left(\mathrm{SO}_{4}\right)_{2}=0.005 \mathrm{M}$ and $\mathrm{HNO}_{3}=0.894 \mathrm{M}$. The curvature is defined as 1/R. S. Maeda et al. Self-walking gel. Adv. Mater. 2007, 19, 3480-3484. Copyright Wiley-VCH Verlag GmbH \& Co.KGaA. Reproduced with permission. (b). Oscillating profiles of the bending-stretching motion for the gel. $l$ is the direct distance between two edges of the curved gel strip at reduced state. $\Delta l$ is the displacement of the direct distance when chemical wave propagates in the gel. S. Maeda et al. Self-walking gel. Adv. Mater. 2007, 19, 3480-3484. Copyright Wiley-VCH Verlag GmbH \& Co.KGaA. Reproduced with permission. (c). Dependence of amplitude (the maximum of displacement, $\Delta l_{\max }$ ) on temperature. S. Maeda et al. Self-walking gel. Adv. Mater. 2007, 19, 3480-3484. Copyright Wiley-VCH Verlag GmbH \& Co.KGaA. Reproduced with permission.

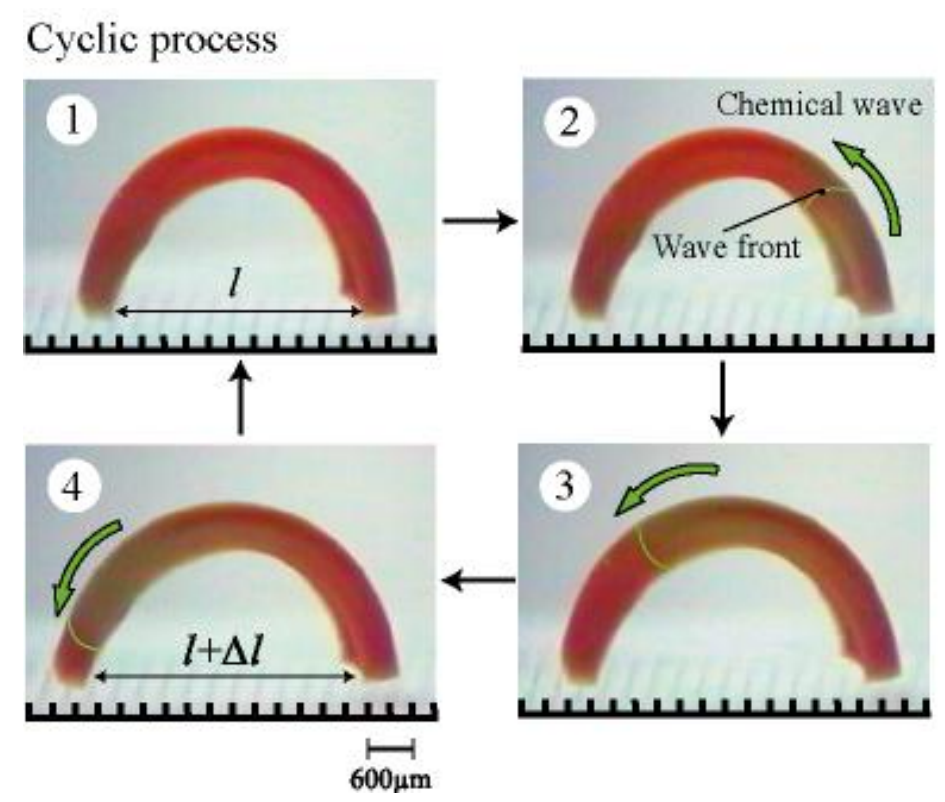

(a)

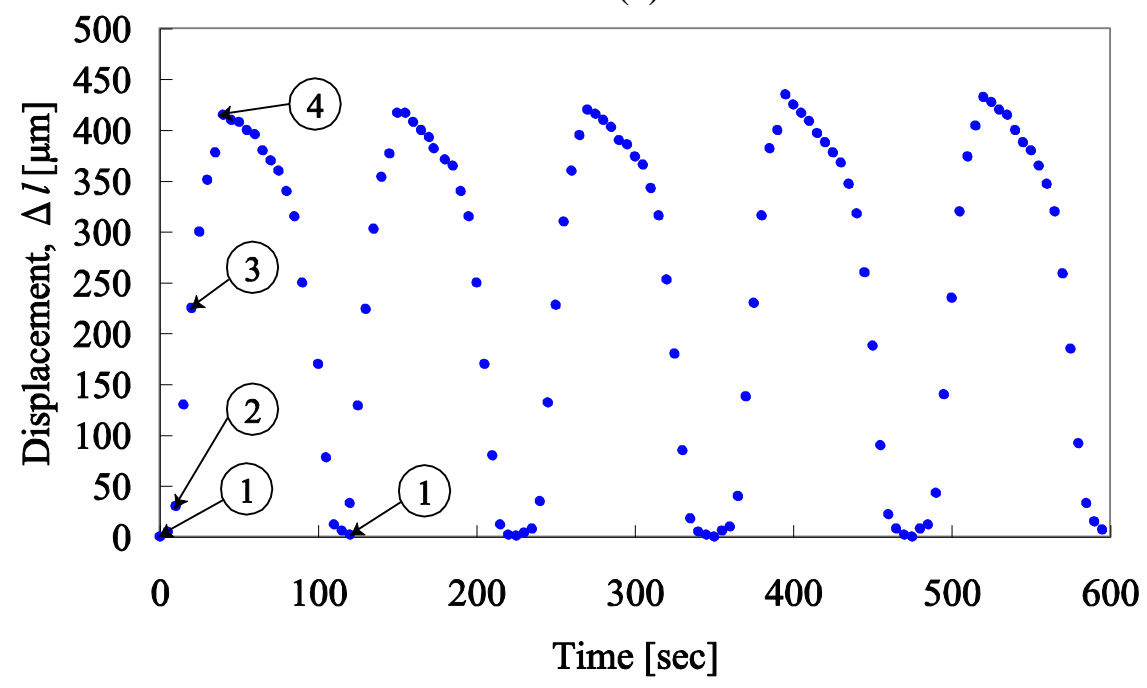

(b) 
Figure 1. Cont.

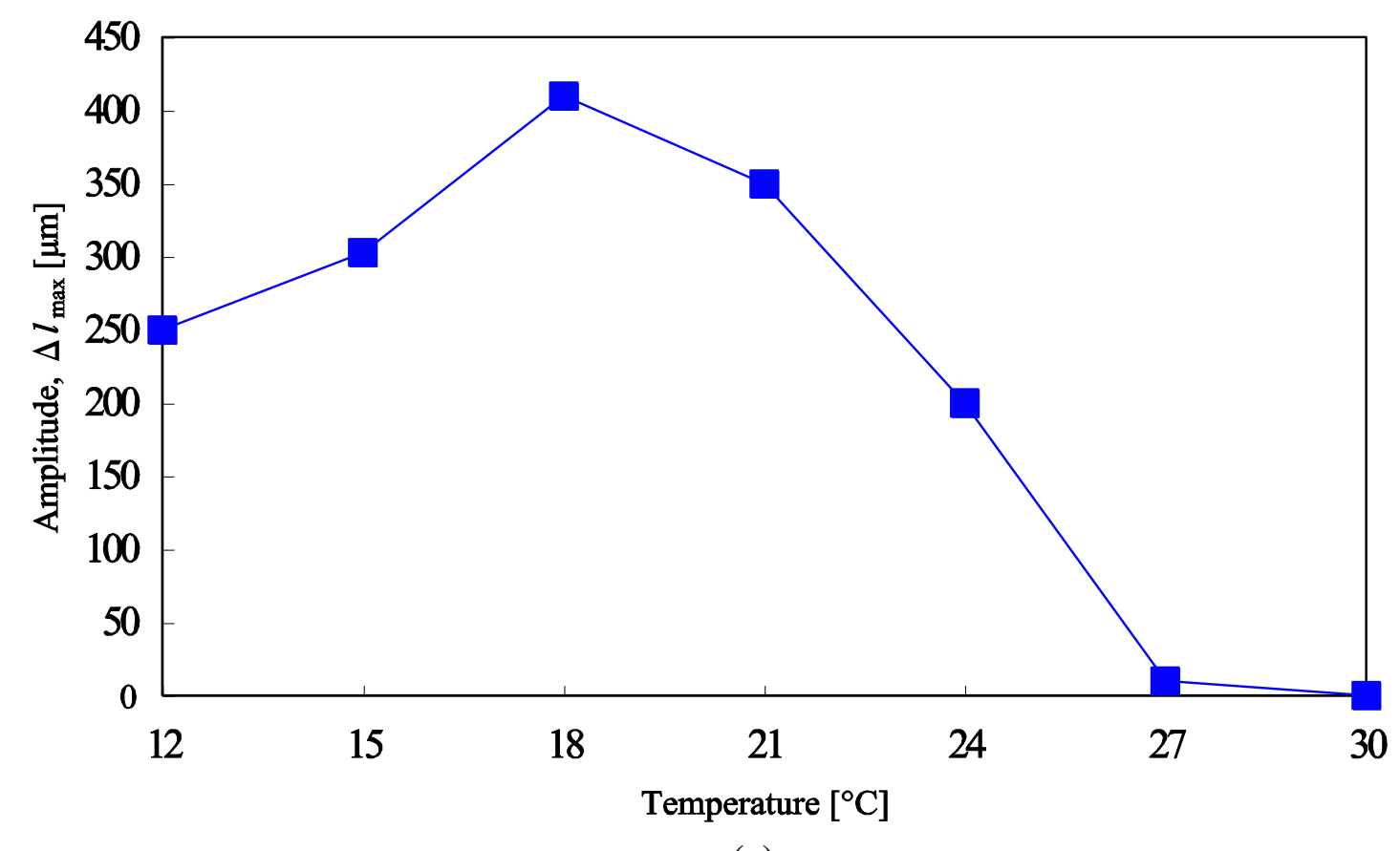

(c)

Figure 2. (a). Surface structure of the ratchet floor made of acrylic sheet. $\Delta$ a stands for the interval of the ratchet teeth. S. Maeda et al. Self-walking gel. Adv. Mater. 2007, 19, 3480-3484. Copyright Wiley-VCH Verlag GmbH \& Co.KGaA. Reproduced with permission. (b). Time course of self-walking motion of the gel actuator (odd number: bending process at the reduced state; even number: stretching process with propagation of chemical wave). During the stretching process, the front edge can slide forward on the floor, but the rear edge is prevented from sliding backwards.Alternatively, during the bending process, the front edge is prevented from going backwards but the rear edge can slide forward. This action is repeated spontaneously, and as a result, the gel walks forward. In one period of the oscillation, the gel can take a step forward by $\Delta$ a. Outer solution: $62.5 \mathrm{mM}$ malonic acid, $84 \mathrm{mM}$ sodium bromate, $0.894 \mathrm{M}$ nitric acid, $18{ }^{\circ} \mathrm{C}$. S. Maeda et al. Self-walking gel. Adv. Mater. 2007, 19, 3480-3484. Copyright Wiley-VCH Verlag GmbH \& Co.KGaA. Reproduced with permission.
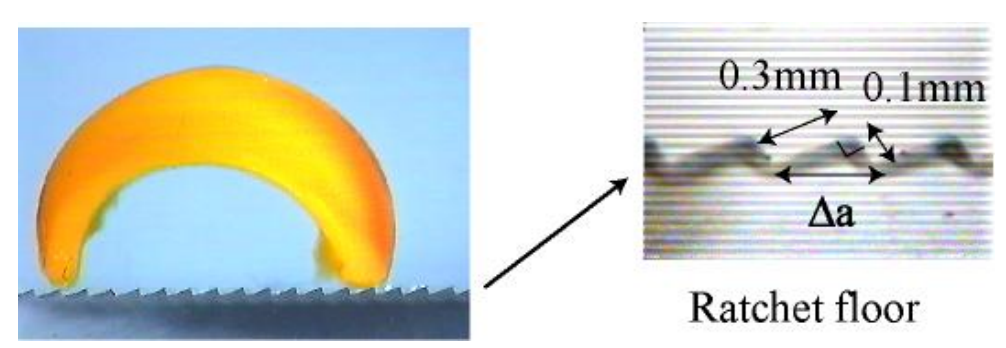

(a) 
Figure 2. Cont.

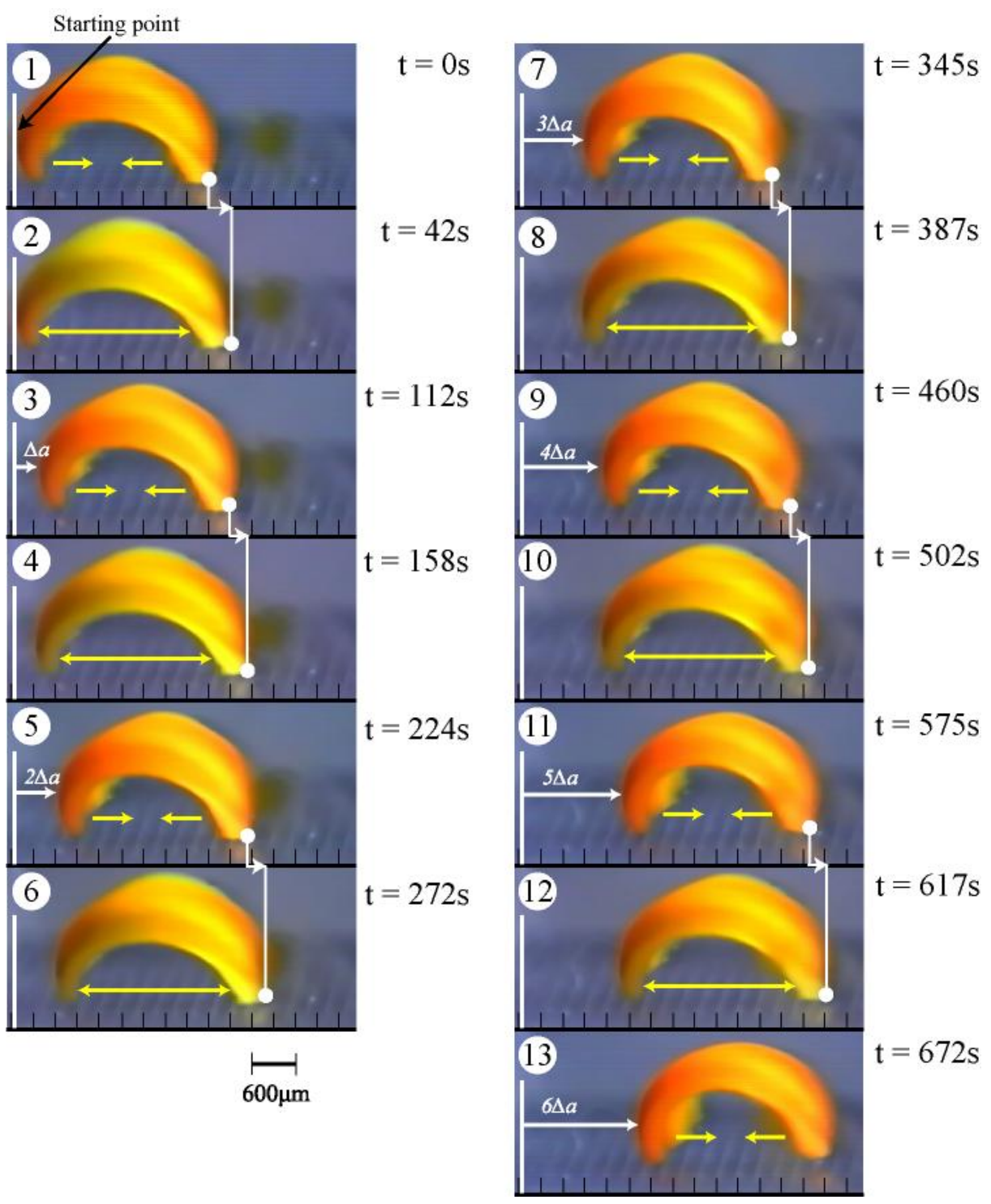

(b)

\section{Design of Peristaltic Motion of Polymer Gel}

Next, we tried to create the peristaltic motion coupled with the chemical wave directly. In previous work, however, it was difficult to observe the peristaltic motion coupled with the chemical wave directly because the mechanical oscillation was too small in comparison to the gel size. Theoretical studies have predicted the occurrence of peristaltic motion within the gel [26,27]. Most recently, we first succeeded in observing the peristaltic motion of the gel [28] directly by utilizing a novel gel with a porous structure. We focus on the kinetics of the polymer gel. The network motion of the gel as given by Tanaka, Hocker and Benedek [29] is

$$
\frac{\partial \vec{u}}{\partial t}=\frac{K+\mu / 3}{f} \operatorname{grad}(\operatorname{div} \vec{u})+\frac{\mu}{f} \Delta \vec{u}
$$

where $\Delta$ denotes the Laplacian and $\vec{u}(\vec{r}, t)$ is the displacement vector that represents the displacement of a point in the network. $K, \mu$ and $f$ are the osmotic bulk modulus, shear modulus of polymer gel and 
friction coefficient between network and fluid medium, respectively. In the case of a radial deformation (1) becomes

$$
\frac{\partial u}{\partial t}=D_{c} \frac{\partial}{\partial r}\left(\frac{\partial u}{\partial r}+2 \frac{u}{r}\right)
$$

with

$$
D_{c}=\frac{K+4 / 3 \mu}{f}
$$

$D_{c}$ is the diffusion coefficient of the polymer gel. If the $D_{c}$ is high value, the response of the gel is fast as shown in Equation (2). In general, the degree of the response of hydro gels composed of chemically cross-linked polymer networks is low because the polymer chains are molecularly restricted by a large number of cross-links. There are remarkable differences in the swelling ratio between the reduced $\mathrm{Ru}(\mathrm{II})$ state and oxidized $\mathrm{Ru}(\mathrm{III})$ state in the poly(NIPAAm-co-Ru(bpy) $)_{3}$ ) gel at the equilibrium swelling state. Actually, the volume oscillation coupled with the redox oscillation of the ruthenium catalyst moiety due to the BZ reaction is very small. The rate of the redox reaction of the Ru moiety is significantly faster than that of the swelling-deswelling of the gel in the equilibrium condition such as mentioned above. Therefore, the poly(NIPAAm-co-Ru(bpy) $)_{3}$ ) self-oscillating gel generated a small mechanical oscillation. In order to produce a large mechanical oscillation in comparison with the gel size, the self-oscillating gel has to first respond to the rate of the BZ reaction. In order to achieve this, we prepared a microphase-separated self-oscillating gel. This was done because in previous work it was reported that the NIPAAm gel with micro scale phase separation underwent quick response [30]. By preparing NIPAAm gel above the lower critical temperature (LCST), the network structure becomes inhomogeneous due to the LCST nature of the NIPAAm component. As a result, the NIPAAm gel forms a porous structure that consists of two regions: polymer rich domains, and the other is aggregations in the matrix of loosely tied network structure. Consequently, rich domains inside the gel clump or loosen rapidly because of an effluent pathway of water due to the porous structure as shown in Figure 3. However, the micro phase separation in the gel strongly depends on the methods and ways of gel preparation. Therefore, control of the phase separation was too difficult by selecting the synthesis temperature. In order to control the micro scale phase separation into the self-oscillating gel, we synthesized the gel under the water-methanol mixture solution by utilizing the hydrophobic casting mold. Generally, in the mixed solvent of water and methanol, the LCST of aqueous PNIPAAm solutions shifts to lower temperature $[31,32]$. So, it is assumed that the micro phase separated structure was introduced inside the gel.

Figure 3. Illustration of the porous gel.

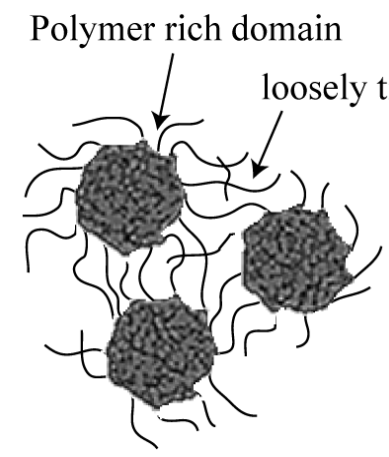

Oxidaized state

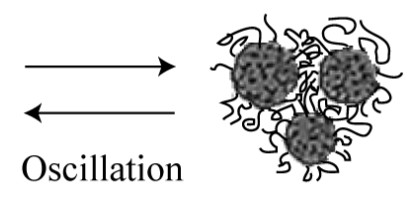

Reduced state 
Figure 4. Relative swelling of microphase-separated self-oscillating gel and poly(NIPAAm-co-Ru(bpy $\left.)_{3}\right)$ gels, $\left(\mathrm{L}_{\mathrm{t}}-\mathrm{L}_{0}\right) /\left(\mathrm{L}_{\mathrm{e}}-\mathrm{L}_{0}\right)$, in the solution of $5 \mathrm{mM} \mathrm{Ce}\left(\mathrm{SO}_{4}\right)_{2}$, $0.894 \mathrm{M} \mathrm{HNO}_{3}$ at $18{ }^{\circ} \mathrm{C}$ as functions of the time $t$ elapsing after changing the solution. $\mathrm{L}_{\mathrm{t}}$, $\mathrm{L}_{0}$ and $\mathrm{L}_{\mathrm{e}}$, are the lengths of the gel at $\mathrm{t}=\mathrm{t}$, initial state and equilibrium state. ( $\square$ ) microphase-separated self-oscillating gel; (ロ) poly(NIPAAm-co-Ru(bpy) $)_{3}$ gel. S. Maeda et al. Peristaltic motion of polymer gels. Angew. Chem. Int. Ed. 2008, 47, 6690-6693. Copyright Wiley-VCH Verlag GmbH \& Co.KGaA. Reproduced with permission.

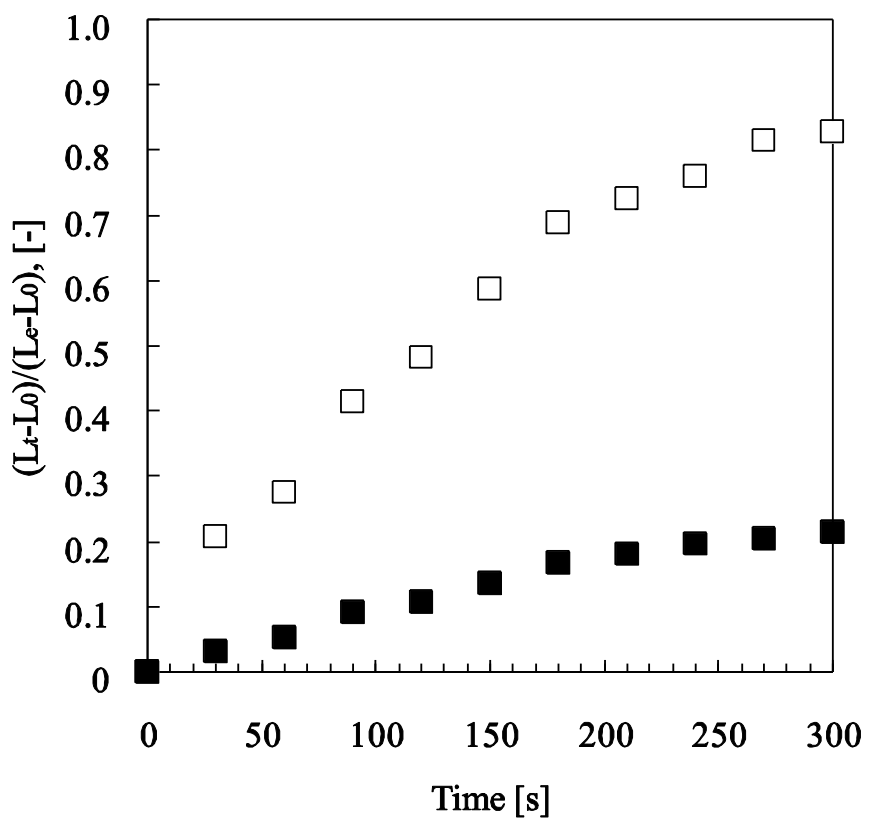

As shown in Figure 4, the swelling speed of the microphase-separated self-oscillating gel was faster than that of the poly(NIPAAm-co-Ru(bpy) $)_{3}$ ) gel at $18{ }^{\circ} \mathrm{C}$. This result indicated that the swelling dynamics of the microphase-separated self-oscillating gel is different from the poly(NIPAAm-co$\mathrm{Ru}(\mathrm{bpy})_{3}$ ) gel. The data supported that the time scale of the swelling kinetics and the chemical reaction matched. This result is significantly important causing a large deformation of the gel by utilizing the BZ reaction. Next, we prepared cubic gel smaller than the wavelength of the chemical wave. Within the miniature gel, the redox change homogeneously occurred without evolution of chemical waves. As for the miniature gel, oscillating profiles of the redox changes, as well as the swelling-deswelling changes, were analyzed by using the image-processing method. Figure 5 shows the self-oscillating behavior of the cubic gel in the aqueous solution containing the three reactants of the BZ reaction (malonic acid, sodium bromate and nitric acid) at constant temperature. The displacement of the mechanical oscillation was around $130 \mu \mathrm{m}$. The displacement of the volume oscillation for the microphase-separated self-oscillating gel is about ten times as large as that for the poly(NIPAAm-co$\mathrm{Ru}(\mathrm{bpy})_{3}$ ) gel. This result indicated that the large mechanical oscillation of the gel required the rapid response to the change in the redox state of the metal catalyst induced by the BZ reaction. From this result, it is expected that gel which is larger than the wavelength of the chemical wave undergoes periodical peristaltic motion when the redox state of the $\mathrm{Ru}(\mathrm{bpy})_{3}$ moiety in the gel periodically change by the $\mathrm{BZ}$ reaction at the constant temperature. 
Figure 5. Oscillating profile of cubic gel. Cubic gel (each side length is about $0.5 \mathrm{~mm}$ ) was immersed in $1 \mathrm{~mL}$ of the mixture solution of the BZ substrates $(62.5 \mathrm{mM}$ malonic acid, $84 \mathrm{mM}$ sodium bromate, $0.894 \mathrm{M}$ nitric acid, $18{ }^{\circ} \mathrm{C}$ ). S. Maeda et al. Peristaltic motion of polymer gels. Angew. Chem. Int. Ed. 2008, 47, 6690-6693. Copyright Wiley-VCH Verlag $\mathrm{GmbH} \& \mathrm{Co.KGaA}$. Reproduced with permission.

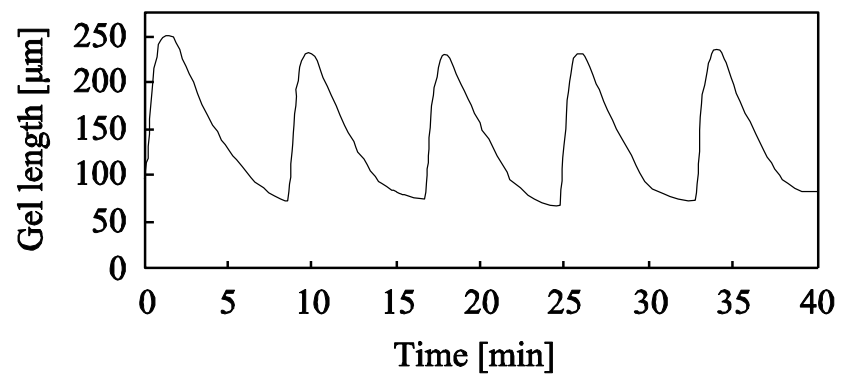

Figure 6. Time course of peristaltic motion of microphase-separated self-oscillating gel in $8 \mathrm{~mL}$ of the mixture solution of the BZ substrates $(62.5 \mathrm{mM}$ malonic acid, $84 \mathrm{mM}$ sodium bromate, $0.894 \mathrm{M}$ nitric acid, $18{ }^{\circ} \mathrm{C}$ ). The green and orange colors correspond to the oxidized and reduced state of $\mathrm{Ru}$ moiety in the gel, respectively. S. Maeda et al. Peristaltic motion of polymer gels. Angew. Chem. Int. Ed. 2008, 47, 6690-6693. Copyright Wiley-VCH Verlag GmbH \& Co.KGaA. Reproduced with permission.
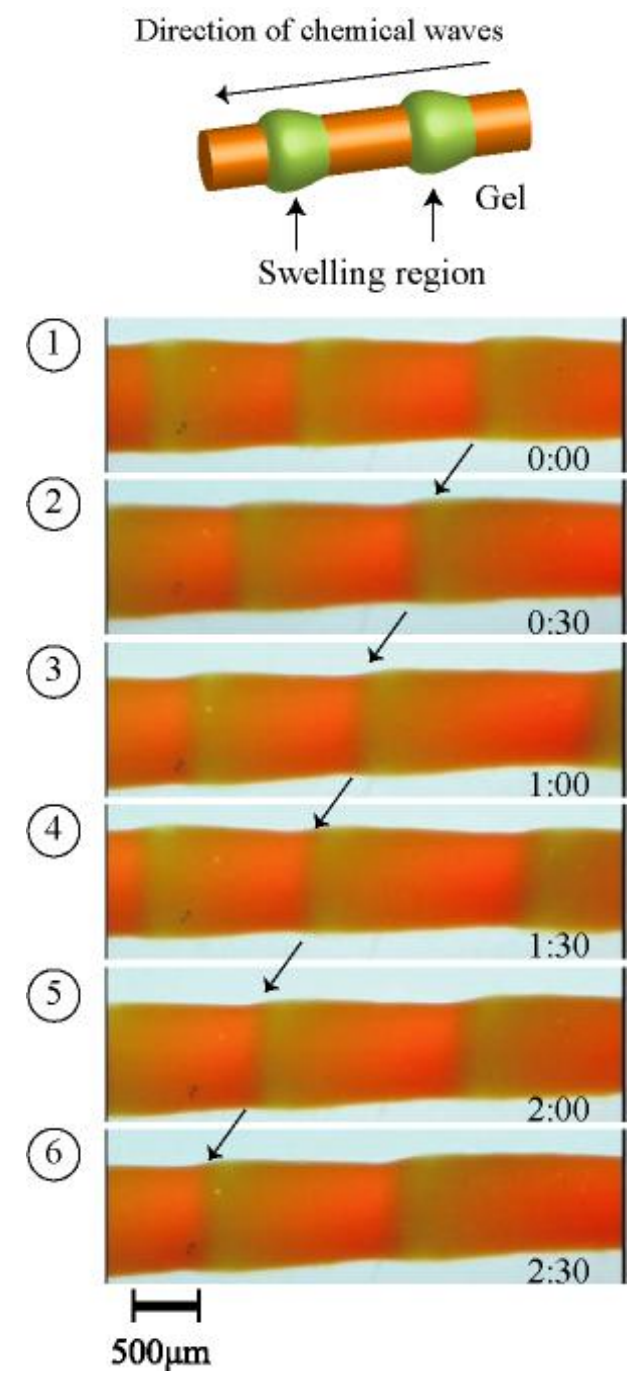
Figure 6 shows the periodical peristaltic motion of the gel driven by the chemical waves of the BZ reaction. We succeeded in directly observing the periodical peristaltic motion of the gel. With the propagation of the chemical waves, the local swelling regions propagated in the gel. This is the first visual evidence of the peristaltic motion of the gel in the macroscopic scale. The shape change of the gel was in excellent agreement with the theory [26,27]. The aspects of the volume change of the gel followed the reaction diffusion dynamics. The chemical wave speed of the BZ reaction was approximately $14.0-30.0 \mu \mathrm{m} / \mathrm{s}$ in the gel.

\section{Matter transport}

Furthermore, we succeeded in conveying the object by utilizing the peristaltic motion of the gel. We set the cylindrical polyacrylamide gel as the object on the rectangular microphase-separated self-oscillating gel in the aqueous solution containing the three reactants of the BZ reaction. Figure 7 illustrates matter transport. The peristaltic surface of the gel pushed and carried the object by rotating it in one direction at about $40 \mu \mathrm{m} / \mathrm{sec}$ by the chemical wave propagation. The gel conveyer carried the object autonomously with millimeter order. It is assumed that the peristaltic motion of the gel can be controlled by changing the concentration of the BZ substrates because the spatiotemporal dynamic pattern changes with changing the outer solution.

Figure 7. Schematic illustration of the matter transport using peristaltic motion of the gel. S. Maeda et al. Peristaltic motion of polymer gels. Angew. Chem. Int. Ed. 2008, 47, 6690-6693. Copyright Wiley-VCH Verlag GmbH \& Co. KGaA. Reproduced with permission.
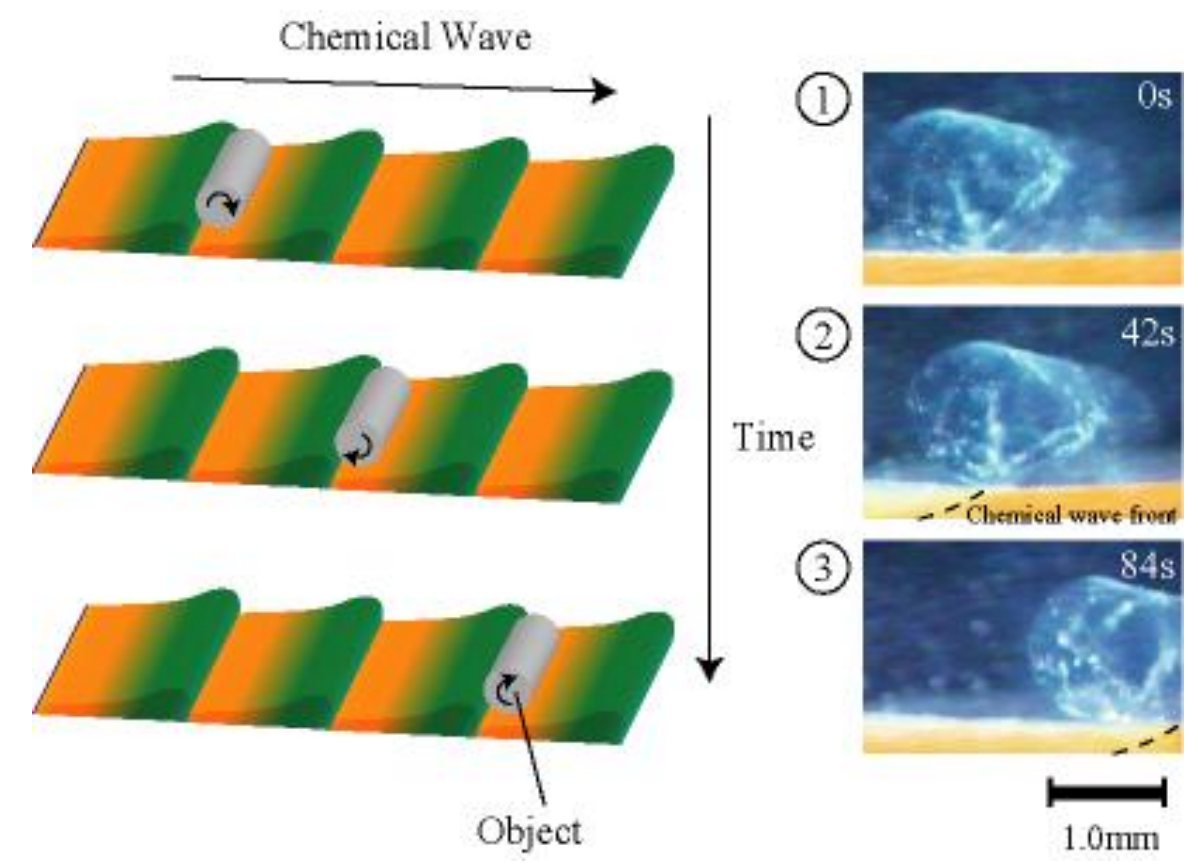

\section{Control of Autonomous Swelling-Deswelling Behavior of a Polymer Gel}

The conventional oscillating gel shrinks at temperatures above the LCST (lower critical solution temperature) because oscillating gel has the thermo sensitive PNIPAAm chain. To realize high-speed 
driving, we attempted to synthesize a novel self-oscillating polymer gel that drives without temperature limitation. In this study, we selected a non-thermo sensitive and biocompatible poly(vinylpyrrolidone) (PVP) as the polymer main chain of the novel self-oscillating gel (poly(VP-co$\mathrm{Ru}(\mathrm{bpy})_{3}$ gel)) [33]. As a result, we first succeeded in causing volume oscillation at a high temperature condition. We studied the influence of the initial concentration of the three BZ substrates other than the metal catalyst and the temperature during the period of self-oscillation as the gel can be controlled by the selection of the initial concentration of the three BZ substrates (malonic acid, sodium bromate, and nitric acid) and the temperature. Moreover, by optimizing the initial concentration of the BZ substrates and the temperature, we succeeded in causing the volume oscillation with frequencies 0.5 Hz. This frequency $(0.5 \mathrm{~Hz})$ of the novel gel was 20 times as large as that of the conventional-type self-oscillating gel (poly(NIPAAmco- Ru(bpy) ${ }_{3}$ gel). We expect that this novel oscillating polymer system will lead to a wide range of applications.

Figure 8 shows the equilibrium swelling behavior of the poly(VP-co-Ru(bpy) $)_{3}$ gels in the $\mathrm{Ce}$ (III) and $\mathrm{Ce}(\mathrm{IV})$ solutions under the same acidic conditions. In the Ce(III) solution, the gel kept a tinge of orange, which indicated that the copolymerized $\mathrm{Ru}(\mathrm{bpy})_{3}$ moiety in the gel was in the reduced state. On the other hand, in the Ce(IV) solution, the gel quickly turned from orange to green, which showed the $\mathrm{Ru}$ (bpy) ${ }_{3}$ moiety in the gel changed the oxidized state from the reduced state. In the oxidized state, the equilibrium volume of the gel was greater than that in the reduced state in all temperature conditions. This is because the solubility of the $\mathrm{Ru}(\mathrm{bpy})_{3}$ moiety has significantly different properties in the oxidized and the reduced states. In the reduced and the oxidized states, there is no observation of the volume phase transition due to the PVP main chain of the gel without LCST.

Figure 8. Equilibrium swelling ratio of poly $\left(\mathrm{VP}-\mathrm{co}-\mathrm{Ru}(\mathrm{bpy})_{3}\right)$ gel in cerium sulfate solutions as a function of temperature. $(\bullet)\left[\mathrm{Ce}_{2}\left(\mathrm{SO}_{4}\right)_{3}\right]=0.001 \mathrm{M}$ and $\left[\mathrm{HNO}_{3}\right]=0.3 \mathrm{M}$; (O) $\left[\mathrm{Ce}\left(\mathrm{SO}_{4}\right)_{2}\right]=0.001 \mathrm{M}$ and $\left[\mathrm{HNO}_{3}\right]=0.3 \mathrm{M}$. The relative length is defined as the ratio of characteristic diameter at the initial state at $20{ }^{\circ} \mathrm{C}$. Reprinted from Ref. [33] with permission; (C) The American Chemical Society.

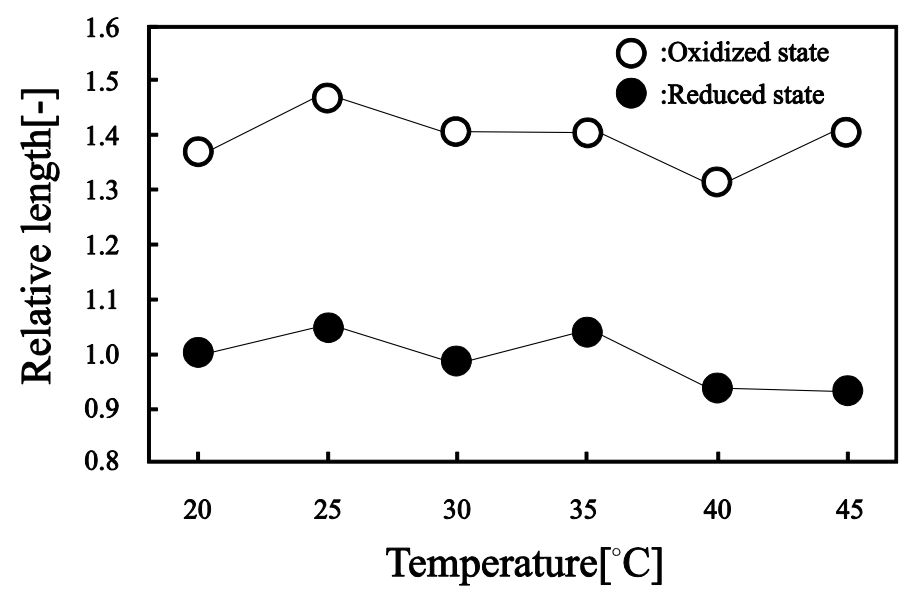

Furthermore, as shown in Figure 9, the period of the swelling-deswelling self-oscillation decreased with increasing the temperature because the temperature affects the $\mathrm{BZ}$ reaction rate in accordance with the Arrenius equation [34]. The maximum frequency $(0.5 \mathrm{~Hz})$ of the poly $\left(\mathrm{VP}-\mathrm{co}-\mathrm{Ru}(\mathrm{bpy})_{3}\right)$ gel was 20 times as large as that of poly(NIPAAm-co-Ru(bpy) $)_{3}$ gel [23]. The self-oscillating behavior of 
the poly $\left(\mathrm{Vp}-\mathrm{co}-\mathrm{Ru}(\mathrm{bpy})_{3}\right)$ gel at $20{ }^{\circ} \mathrm{C}$ and $50{ }^{\circ} \mathrm{C}$ is shown in Figure 9(b) and 9(c), respectively. The displacement of the volume change self-oscillation at $20{ }^{\circ} \mathrm{C}$ and $50{ }^{\circ} \mathrm{C}$ were about $10 \mu \mathrm{m}$ and $4 \mu \mathrm{m}$, respectively. These results clarified that the displacement of the swelling-deswelling self-oscillation of the gel has a trade-off relationship with the period of self-oscillation; that is, the length of the volume change decreased on increasing the period. Therefore, we are investigating a corrective strategy for the trade-off relationship between the period of self-oscillation and the displacement of the gel, in order to realize autonomous soft actuators that cause the large deformation at a high speed.

Figure 9. (a) Dependence of the self-oscillation period on the temperature. $(\bullet)$ plots and (०) plots show the linear relation and the saturated line vs. temperature, respectively. (b) Self-oscillating profile of cubic poly(VP-co-Ru(bpy) $\left.)_{3}\right)$ gel at $50{ }^{\circ} \mathrm{C}(\mathrm{MA}=0.08 \mathrm{M}$, $\mathrm{NaBrO}_{3}=0.48 \mathrm{M}$ and $\mathrm{HNO}_{3}=0.48 \mathrm{M}$ ). (c) Self-oscillating profile of cubic poly(VP-co$\left.\mathrm{Ru}(\text { bpy })_{3}\right)$ gel at $20{ }^{\circ} \mathrm{C}\left(\mathrm{MA}=0.08 \mathrm{M}, \mathrm{NaBrO}_{3}=0.48 \mathrm{M}\right.$ and $\left.\mathrm{HNO}_{3}=0.48 \mathrm{M}\right)$. Cubic gel (each side length is about $2 \mathrm{~mm}$ and $20 \mathrm{~mm}$ ) was immersed in $8 \mathrm{~mL}$ of the mixture solution of the BZ substrates. Reprinted from Ref. [33] with permission; (C) The American Chemical Society.

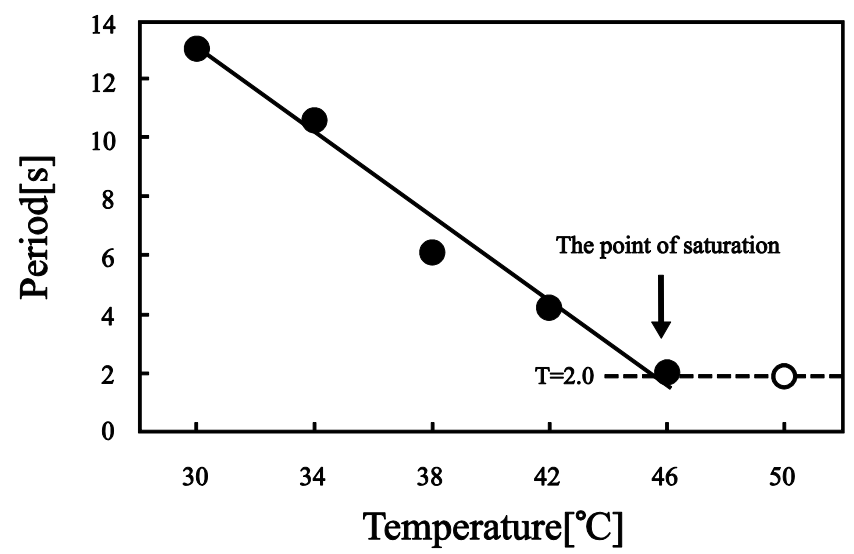

(a)

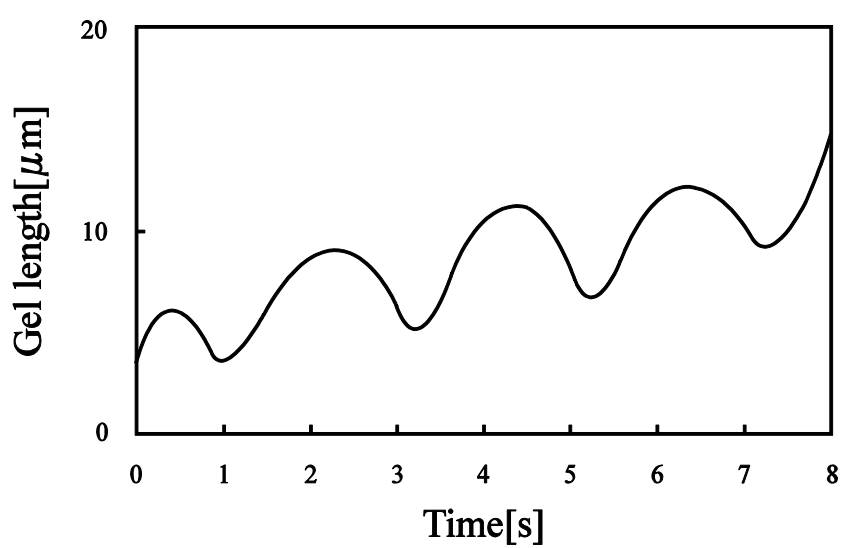

(b)

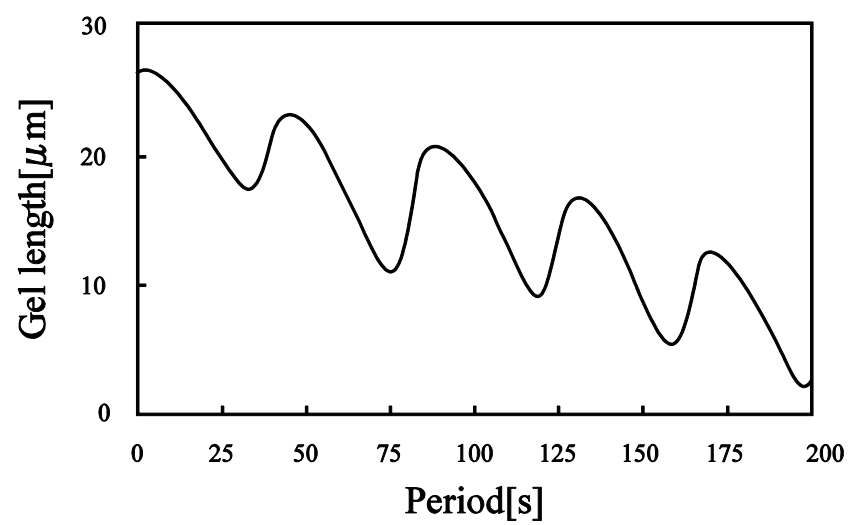

(c)

\section{Conclusion}

In this paper, we introduced novel autonomous polymer gel actuators driven by chemical energy. We consider autonomous gel actuators to be the preliminary step for "Chemical Robotics". Chemical robots differ from mechanical robots in terms of assembly, material, driving force, etc. At the present 
stage, an operating condition for the walking motion is limited to one dimension. Our next work is will develop various gel motions caused by the reaction diffusion system.

\section{Acknowledgements}

This work was supported in part by: (1) The Global COE (Centers of Excellence) Program, "Global Robot Academia", Waseda University; (2) "Establishment of Consolidated Research Institute for Advanced Science and Medical Care," Encouraging Development Strategic Research Centers Program, the Special Coordination Funds for Promoting Science and Technology, Ministry of Education, Culture, Sports, Science and Technology, Japan; (3) Grant-in-Aid for Young Scientists (B) (21750222); and (4) Grant-in-Aid for Tokyo Ouka. The authors gratefully thank all collaborators in this research project.

\section{References}

1. Hirokawa, Y.; Tanaka, T. Volume Phase Transition in a Non Ionic Gel. J. Chem. Phys. 1984, 81, 6379-6380.

2. Tanaka, T. Collapse of Gels and Critical Endpoint. Phys. Rev. Lett. 1978, 40, 820-823.

3. Suzuki, A.; Tanaka, T. Phase Transition in a Polymer Gels Induced by Visible-Light. Nature 1990, 346, 345-347.

4. Ilmain, F.; Tanaka, T.; Kokufuta, E.; Volume Transition in a Gel by Hydrogen Bonding. Nature 1991, 349, 400-401.

5. Tanaka, T.; Nishio, I.; Sun, S.T.; Uenoshio, S.; Collapse of Gels in an Electric Field. Science 1982, 218, 467-469.

6. Otake, M.; Inaba, M.; Inoue, H.; Kinematics of Gel Robots Made of Electro-Active Polymer PAMPS Gel. In Proceedings of the IEEE International Conference on Robotics and Automation, San Francisco, CA, USA, April 2000; pp. 488-493.

7. Kwon, G.H.; Park, J.Y.; Kim, J.Y.; Frisk, M.L.; Beebe, D.J.; Lee, S.H. Biomimetic Soft Multifunctional Miniature Aquabots. Small 2008, 4, 2148-2153.

8. Osada, Y.; Okuzaki, H.; Hori, H. A Polymer Gel with Electrically Driven Motility. Nature 1992, 355, 242-244.

9. Tanaka, T. Gels. Sci. Am. 1981, 244, 110-116.

10. Yoshida, R.; Sakai, K.; Okano, T.; Sakurai, Y. Modern Hydrogel Delivery Systems. Adv. Drug Delivery Rev. 1993, 11, 85-108.

11. Hu, Z.; Zhang, X.; Li, Y. Synthesis and Application of Modulated Polymer Gels. Science 1995, 269, 525-527.

12. Yeghiazarian, L.; Mahajan, S.; Montemagno, C.; Cohen, C.; Wiesner, U. Directed Motion and Cargo Transport through Propagation of Polymer-Gel Volume Phase Transitions. Adv. Mater. 2005, 17, 1869-1873.

13. Sidorenko, A.; Krupenkin, T.; Taylor, A.; Fratzl, P.; Aizenberg, J. Reversible Switching of Hydrogel-Actuated Nanostructures into Complex Micropatterns. Science 2007, 315, 487-490. 
14. Feinberg, A.W.; Feigel, A.; Shevkoplyas, S.S.; Sheehy, S.; Whitesides, G.M.; Parker, K.K. Muscular Thin Films for Building Actuators and Powering Devices. Science 2007, 317, 1366-1370.

15. Crook, C.J.; Smith, A.; Jones, A.L.; Ryan, A.J. Chemically Induced Oscillations in a pH-Responsive Hydrogel. Phys. Chem. Chem. Phys. 2002, 4, 1367-1369.

16. Lerox, J.C.; Siegel, R.A. Autonomous Gel/Enzyme Oscillator Fueled by Glucose: Preliminary Evidence for Oscillations. Chaos 1999, 9, 267-275.

17. Labrot, V.; De Kepper, P.; Boissonade, J.; Szalai, I.; Gauffre, F. Wave Patterns Driven by Chemomechanical Instabilities in Responsive Gels. J. Phys. Chem. B 2005, 109, 21476-21480.

18. Zaikin, A.N.; Zhabotinsky, A.M.; Concentration Wave Propagation in Two-dimensional Liquid-Phase Self-Oscillating System. Nature 1970, 225, 535-537.

19. Noszticzius, Z.; Horsthemke, W.; Mccormick, W.D.; Swinney, H.L.; Tam, W.Y. Sustained Chemical Waves in an Annular Gel Reactor: A Chemical Pinwheel. Nature 1987, 329, 619-621.

20. Yamaguchi, T.; Kuhnert, L.; Nagy-Ungvarai, Z.S.; Muller, S.C.; Hess, B. Gel Systems for the Belousov-Zhabotinskii Reaction. J. Phys. Chem. 1991, 95, 5831-5837.

21. Ishiwatari, T.; Kawaguchi, M.; Mitsuishi, M. Oscillatry Reactions in Polymer Systems. J. Polym. Sci. A Polm. Chem. 1984, 22, 2699-2704.

22. Yoshida, R.; Sakai, T.; Ito, S.; Yamaguchi, T. Self-Oscillation of Polymer Chains with Rhythmical Soluble-Insoluble Changes. J. Am. Chem. Sci. 2002, 124, 8095-8098.

23. Yoshida, R.; Takahashi, T.; Yamaguchi, T.; Ichijo, H. Self-Oscillating Gel. J. Am. Chem. Soc. 1996, 118, 5134-5135.

24. Maeda, S.; Hara, Y.; Yoshida, R.; Hashimoto, S. Control of the Dynamic Motion of a Gel Actuator Driven by the Belousov-Zhabotinsky Reaction. Macromol. Rapid. Commun. 2008, 29, 401-405.

25. Maeda, S.; Hara, Y.; Sakai. T.; Yoshida, R.; Hashimoto, S. Self-Walking Gel. Adv. Mater. 2007, 19, 3480-3484.

26. Yashin, V.V.; Balazs, A.C. Pattern Formation and Shape Changes in Self-Oscillating Polymer Gels. Science 2006, 314, 798-801.

27. Yashin, V.V.; Balazs, A.C. Theoretical and Computational Modeling of Self-Oscillating Polymer Gels. J. Chem. Phys. 2007, 126, 124707.

28. Maeda, S.; Hara, Y.; Yoshida, R.; Hashimoto, S. Peristaltic Motion of Polymer Gels. Angew. Chem. Int. Ed. 2008, 47, 6690-6693.

29. Tanaka, T.; Hocker, L.; Benedek, G,B. Spectrum of Light Scattered from a Viscoelastic Gel. $J$. Chem. Phys. 1973, 53, 5151-5159.

30. Kabra, B.G.; Gehrke, S.H. Synthesis of Fast Response, Temperature-Sensitive Poly(N-isopropylacrylamide) Gels. Polym. Commun. 1991, 32, 322-323.

31. Hirotsu, S. Phase Transition of a Polymer Gel in Pure and Mixed Solvent Media. J. Phys. Soc. Jpn. 1986, 56, 233-242.

32. Tanaka, F.; Koga, T.; Kojima, H.; Winnik, M.F. Temperature- and Tension-Induced Coil-Globule Transition of Poly( $N$-isopropylacrylamide) Chains in Water and Mixed Solvent of Water/Methanol. Macromolecules 2009, 42, 1321-1330. 
33. Nakamaru, S.; Maeda, S.; Hara, Y.; Hashimoto, S. Control of Autonomous Swelling-Deswelling Behavior for a Polymer gel. J. Phys. Chem. B 2009, 113, 4609-4613.

34. Yoshikawa, K. Distinct Activation Energies for Temporal and Spatial Oscillations in the Belousov-Zhabotinskii Reaction. Bull. Chem. Soc. Jpn. 1982, 55, 2042.

(C) 2011 by the authors; licensee MDPI, Basel, Switzerland. This article is an open access article distributed under the terms and conditions of the Creative Commons Attribution license (http://creativecommons.org/licenses/by/3.0/). 